\title{
A NEW LEPTOTHORAX FROM ALABAMA (HYMENOPTERA: FORMICIDAE) ${ }^{1}$
}

\author{
By Edward O. Wilson \\ University of Alabama
}

\section{Leptothorax (Myrafant) tuscaloosae, new species}

Holotype worker. - Total length approximately $1.9 \mathrm{~mm}$; length of alitrunk, measured from the dorsal base of the pronotal collar to the tip of the posterior propodeal flange, $0.554 \mathrm{~mm}$.; length of head, measured in profile from the anterior margin of the clypeus to the extreme occipital border, $0.512 \mathrm{~mm}$.; cephalic index 93.5. (All measurements except total length given in this description with a maximum error of $\pm 0.016 \mathrm{~mm}$.)

Eye oval, moderately prominent, with nine ocelli across its greatest length, located nearer the anterior than the posterior border of the head. Head subrectangular, with weakly convex posterior border, rounded posterior angles, and weakly convex, subparallel sides. Clypeus depressed, 1.2 times longer than broad, its anterior border rounded and entire. Antenna eleven-segmented; scape failing by approximately its greatest width to meet the occipital angle; funicular club three-jointed, as long as the remainder of the funiculus, the apical segment longer than the preceding two combined. Mandible with five teeth, the apical tooth the largest. Alitrunk slender, seen from above $0.272 \mathrm{~mm}$. across its greatest width at the pronotum, evenly arcuate in profile, sloping to the base of the propodeal spines; humeri well rounded; pro-mesonotal and mesoepinotal sutures absent; other thoracic sutures weak or absent. Propodeal spines slender and acute, slightly and gradually curved inward and downward, approximately as long as the declivious face of the propodeum, the basal portions in profile forming an angle of approximately $120^{\circ}$ with the basal face of the propodeum, their bases $0.096 \mathrm{~mm}$. apart. Femora and tibiae noticeably incrassated. Petiolar node in profile with anterior face concave, and meeting the dorsal face in a bluntly rounded angle. Dorsum of postpetiole $\mathbf{1 . 5}$ times broader than long, not constricted in posterior half, with subparallel sides.

${ }^{1}$ Published with a grant from the Museum of Comparative Zoology at Harvard College. 
Mandibles covered dorsally by close-set, longitiudinal striae. Clypeus with a dark median carina running from anterior to posterior border; the remainder of the clypeus more or less longitudinally rugulose. Frontal area, frontal lobes, and cheeks rugulose. Remainder of head, most of the thorax, and the gaster moderately shining, with extremely fine punctures. Propodeum and meso- and metasternal regions of thorax rugulose, the rugulae of the declivious face of the propodeum transverse. Petiole and postpetiole densely and coarsely granulose.

Body covered by moderately abundant, long, coarse, grayish, erect hairs. Antennae with moderately abundant, short, very fine hairs over entire surface; many of these hairs on funiculus and a small number on scape suberect to erect but the majority appressed. Legs with a sparse growth of hairs similar to those on antennae; most of these appressed by a scattered few suberect to erect.

Body dark brown; mandibles, antennae, and legs pale yellow; femora infuscated over entire surface except for ends.

Gynetype. - Differing from the worker in the usual characters separating these two phases. Total length approximately $2.7 \mathrm{~mm}$., length of alitrunk $0.912 \mathrm{~mm}$., greatest width of alitrunk $0.576 \mathrm{~mm}$., length of head $0.568 \mathrm{~mm}$., cephalic index 100. Propodeal spines short and robust, their length less than the distance between their bases and approximately half the length of the declivious face of the propodeum. Sculpturing, pilosity, and color essentially the same as in the worker.

Type locality. - Tuscaloosa, Alabama.

The holotype worker, the gynetype, and forty-two paratypes consisting of seven dealate queens and thirty-five workers were collected by the author August 20, 1947, in a small patch of woods directly north of Guthrie's Nursery, which is at the Tuscaloosa Memorial Cemetery near the outskirts of the city.

Additional locality: Elrod, Tuscaloosa Co., Ala. Thirteen paratypes consisting of two dealate queens and eleven workers were collected by Barry D. Valentine and the author May 10, 1949, in the Sipsey River swamp several miles east of the town.

The paratypes of this small species vary from the holotype and gynetype noticeably in color and size. Callows are pale yellow, with infuscated head, gaster, and femora. The other specimens in the type series vary from medium to dark, almost piceous, brown. The mandibles, antennae, and legs of all are pale yellow, the femora 
infuscated. Total length in the workers varies (approximately) from $1.9 \mathrm{~mm}$. to $2.1 \mathrm{~mm}$.; length of alitrunk varies from $0.554 \mathrm{~mm}$. to $0.684 \mathrm{~mm}$.; with a mean of $0.609 \mathrm{~mm}$.; length of head varies from $0.505 \mathrm{~mm}$. to $0.570 \mathrm{~mm}$., with a mean of $0.530 \mathrm{~mm}$. Total length in the queens does not possess variability sufficient for measurement, which is somewhat dependent on the degree of distention in the gaster; length of alitrunk varies from $0.929 \mathrm{~mm}$. to $0.962 \mathrm{~mm}$., with a mean of $0.942 \mathrm{~mm}$; length of head varies from $0.570 \mathrm{~mm}$. to $0.603 \mathrm{~mm}$., with a mean of $0.590 \mathrm{~mm}$. The workers from Elrod are notably larger on the average than the ones from Tuscaloosa: mean length of alitrunk $0.649 \mathrm{~mm}$. as opposed to $0.600 \mathrm{~mm}$., mean length of head $0.549 \mathrm{~mm}$. as opposed to $0.524 \mathrm{~mm}$.

The holotype, the gynetype, and twelve paratypes have been deposited in the United States National Museum under U. S. N. M. No. 60339. Paratypes are in the collections of the author, the Museum of Comparative Zoology at Cambridge, and the University of Alabama.

Dr. M. R. Smith has very kindly examined types of the new species and has expressed the opinion that its closest morphological affinities are apparently to Leptothorax (Myrafant) curvispinosus Mayr. It can be distinguished from that species by its differently shaped propodeal spines and petiole, its smaller size, much darker body coloration, and feebler cephalic and thoracic sculpturing. Because of the shining dorsal surface of the head, the new species runs down to L. (M.) longispinosus Roger in W. S. Creighton's recent key of the North American Leptothorax (The Ants of North America, Bull. M. C. Z., Vol. 104). It can be distinguished from longispinosus by its smaller size, different coloration (mandibles, antennae, and legs in longispinosus moderately brown), feeble thoracic sculpturing, and shorter and more elevated propodeal spines (the spines of longispinosus form an angle of nearly $180^{\circ}$ with the dorsum of the propodeum when viewed in profile).

The Tuscaloosa colony was found in a small cavity in a bank of earth under a bed of moss. It was at the base of a large oak in an open area fringing a bay-gum swamp. The Elrod colony was found in a small cavity in the earth covered partly by an overhanging root and party by thin leaf litter. It was in a densely shaded area also on the fringes of a bay-gum swamp. Stray workers were taken during the day on low bushes near both nests. 

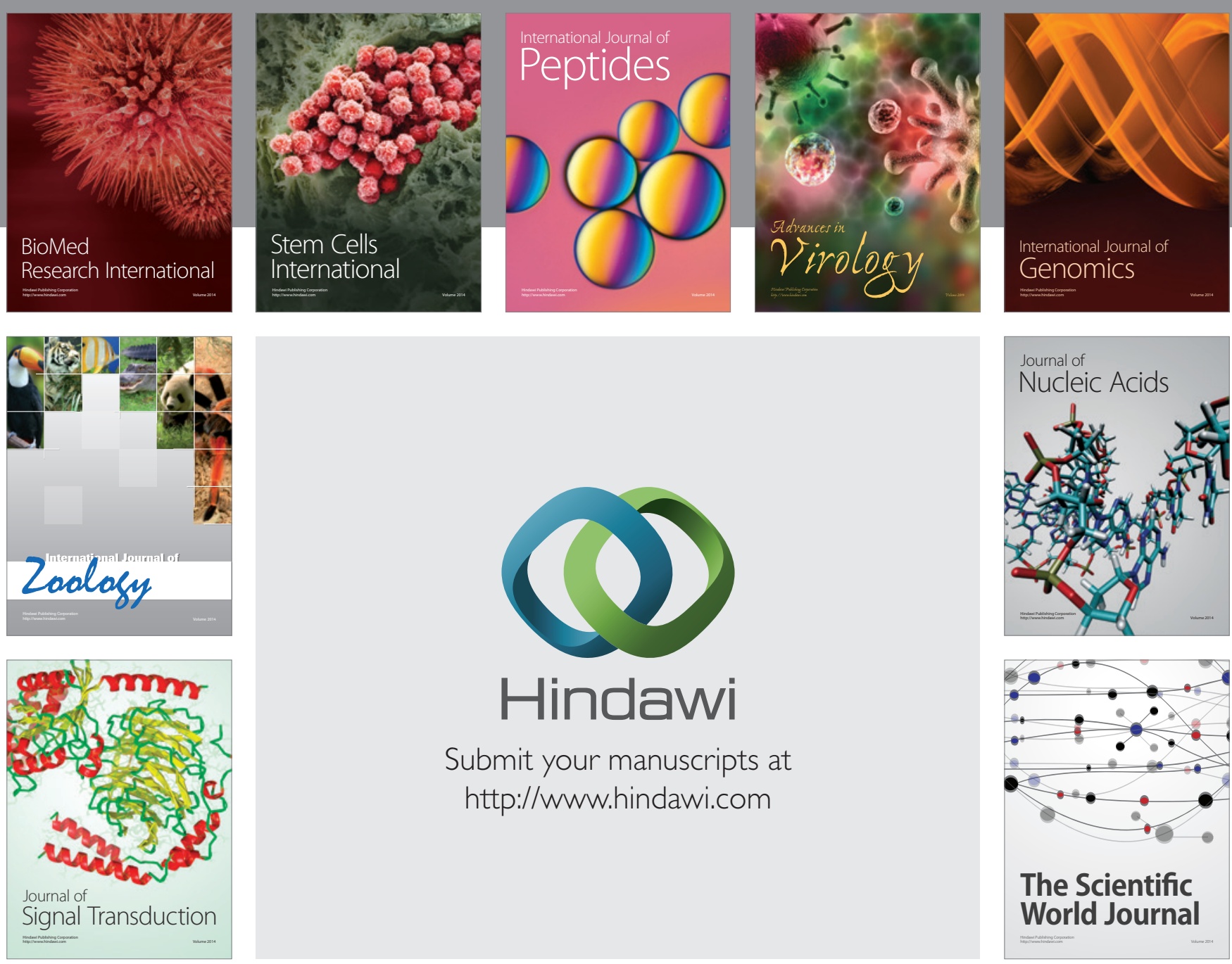

Submit your manuscripts at

http://www.hindawi.com
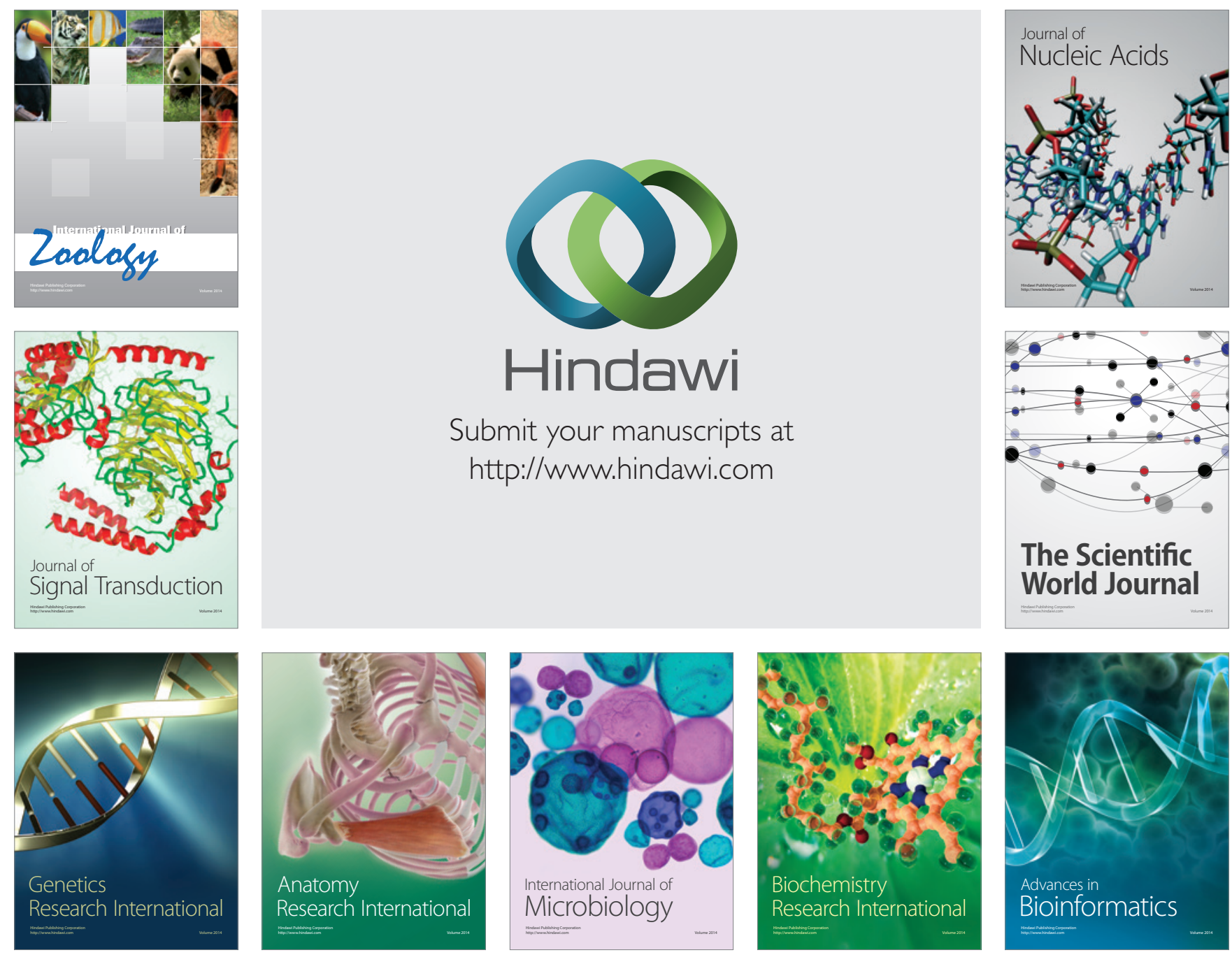

The Scientific World Journal
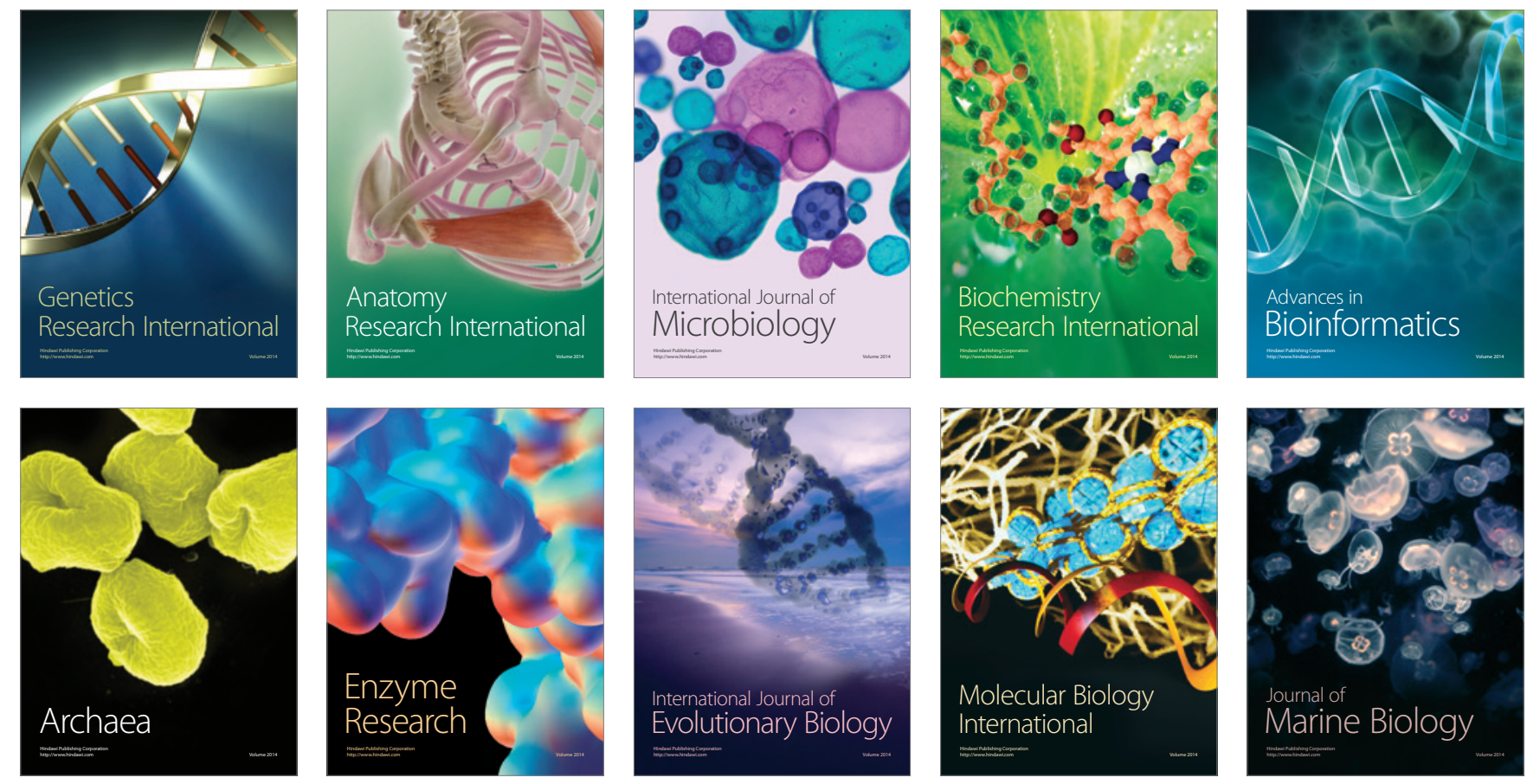